\title{
A family with Robertsonian translocation: a potential mechanism of speciation in humans
}

\author{
Jieping Song ${ }^{1 \dagger}, \mathrm{Xi} \mathrm{li}^{2 \dagger}$, Lei Sun ${ }^{3 \dagger}$, Shuqin Xu', Nian Liu', Yanyi Yao ${ }^{1}$, Zhi Liu', Weipeng Wang ${ }^{1}$, Han Rong ${ }^{4 *}$
} and Bo Wang ${ }^{1 *}$

\begin{abstract}
Background: Robertsonian translocations occur in approximately one in every 1000 newborns. Although most Robertsonian translocation carriers are healthy and have a normal lifespan, they are at increased risk of spontaneous abortions and risk of producing unbalanced gametes and, therefore unbalanced offspring. Here we reported a previously undescribed Robertsonian translocation.

Case Presentation: We identified three Robertsonian translocation carriers in this family. Two were heterozygous translocation carriers of $45, \mathrm{XX}$ or $\mathrm{XY}, \operatorname{der}(14 ; 15)(\mathrm{q} 10 ; \mathrm{q} 10)$ and their son was a homozygous translocation carrier of a 44,XY,der(14;15)(q10;q10), der(14;15)(q10;q10) karyotype. Chromosomal analysis of sperm showed $99.7 \%$ of sperm from the homozygous translocation carrier were normal/balanced while only $79.9 \%$ of sperm from the heterozygous translocation carrier were normal/balanced. There was a significantly higher frequency of aneuploidy for sex chromosome in the heterozygous translocation carrier.
\end{abstract}

Conclusions: The reproductive fitness of Robertsonian translocation carriers is reduced. Robertsonian translocation homozygosity can be a potential speciation in humans with 44 chromosomes.

Keywords: Chromosome karyotype, FISH, aCGH, Robertsonian translocation homozygosity, Evolution

\section{Background}

Robertsonian translocations, fusions between two acrocentric chromosomes, are the most common structural chromosomal rearrangements in humans and occur in approximately 1 in every 1000 newborns [1]. It occurs in the five acrocentric chromosomes, 13, 14, 15, 21, and 22, which have very small short arms that contain no unique genes. During a Robertsonian translocation, the participating chromosomes break at their centromeres and the long arms fuse to form a single chromosome with a single centromere. The short arms also join to form a smaller reciprocal product, which typically contains nonessential genes and is usually lost within a few cell

\footnotetext{
*Correspondence: 89657471@qq.com; wangbo1005@163.com

${ }^{\dagger}$ Equal contributors

${ }^{4}$ Shenzhen mental health center, Shenzhen Kangning Hospital, Shenzhen, Guangdong, People's Republic of China

'Genetics Laboratory, Hubei Maternal and Child Health Hospital, Wuhan,

Hubei, People's Republic of China

Full list of author information is available at the end of the article
}

divisions. This type of translocation is cytologically visible and can reduce chromosome number if the smaller chromosome that results from a translocation is lost over cellular divisions. However, the smaller chromosome lost may carry so few genes that it can be lost without an ill effect to the individual $[2,3]$.

When the translocation is balanced, the person with it is called a Robertsonian translocation carrier. As carriers are healthy and have a normal lifespanand, many never discover their unusual chromosome rearrangement. Therefore, the translocation can be passed down in families for many generations without anyone discovering. Robertsonian translocation carriers produce six types of gametes. At the end of meiosis I, segregation of the translocated and nontranslocated chromosomes from the two different chromosome pairs involved leads to the formation of either balanced gametes via alternate segregation or unbalanced gametes via adjacent segregation during anaphase $[1,2]$. Meiotic tetravalent configuration tends to segregate 
in alternate way, resulting in preferential production of normal/balanced spermatozoa. However, certain percentages of unbalanced gametes derived from adjacent segregation are also produced, leading to the increased risk of miscarriage and chromosomally unbalanced fetus. In this study, we investigated a Robertsonian translocation family and analyzed the meiotic segregation patterns and interchromosomal effects in sperm from two Robertsonian translocation carriers.

\section{Case presentation}

\section{Clinical information}

A 25-year-old Chinese man (IV-1) married to a nonconsanguineous woman with normal chromosomes (IV-2). This couple had a son (V-1) who died at 6-month old without an autopsy. Previous chromosome analysis indicated V1 had a 45,XY,der(14;15)(q10;q10) karyotype. The parents of the propositus (IV-1) are phenotypically normal first cousins (III-1, III -2). Their parents, a mutual uncle and both grandparents were not available for cytogenetic analysis (Fig. 1). Mental state examination was performed on IV-1 by a psychiatrist.

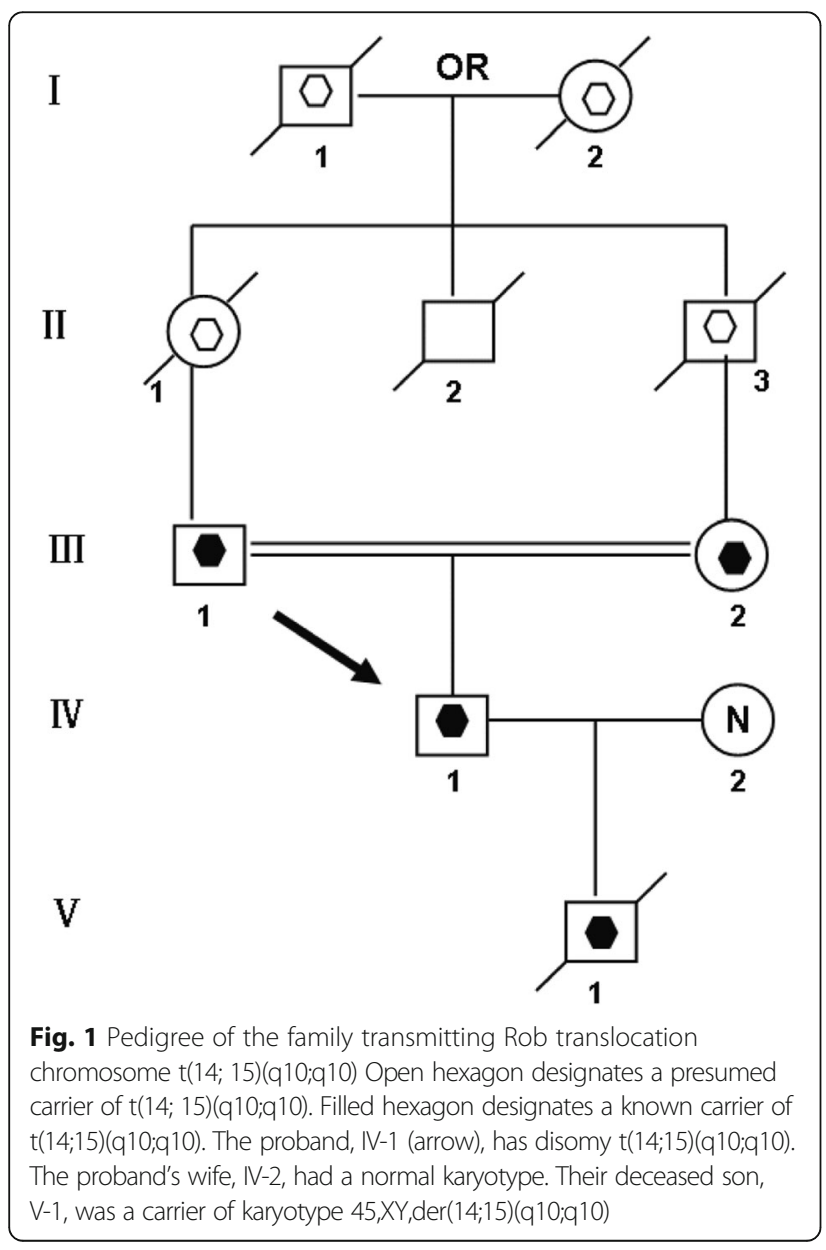

\section{Karyotype analysis}

G-banding karyotype analysis was performed on all the four members in the family. The previously undescribed karyotype, 44,XY,der(14;15)(q10;q10), der(14;15)(q10;q10), was further authenticated by the Chinese Academic Committee of the State Key Laboratory of Medical Genetics (Fig. 2).

\section{Fluorescence in situ hybridization (FISH)}

Sperm chromosomes from patient III-1 and IV-1 were analyzed by FISH. Semen samples were assessed for volume, concentration and motility according to the World Health Organization criteria. After semen analysis, progressively mobile sperm were isolated and washed twice in phosphate buffered saline $(\mathrm{pH} 7.4)$ and centrifuged at $1500 \mathrm{rpm}$ for $11 \mathrm{~min}$. Pellets were fixed with $5 \mathrm{ml}$ of acetic acid/methanol mixture (1:3) for at least $30 \mathrm{~min}$ at $4{ }^{\circ} \mathrm{C}$. Aliquots $(40-50 \mu \mathrm{l})$ of the resulting suspension of nuclei were smeared on cold pre-cleaned slides. Nuclei decondensation was performed in $1 \mathrm{~N} \mathrm{NaOH}$ for 2 min. The slides were then degraded by a graded ethanol series (70 \%, $90 \%, 100 \%$ ) and denatured with $0.25 \%$ formamide in 2xSSC followed by overnight hybridization with a combination of commercially available probes. Two sets of probe mixtures were used in this study. Dual-color FISH was carried out using locusspecific probes (LSP) and Tel probes from Vysis (Vysis, Downers Grove, IL, USA). For patient IV-1 and III-1,TelVysion Probe 14q (D14S1420, Spectrum Red) was used for 14q32.33 and TelVysion Probe 15q(D15S120, Spectrum Green) was used for $15 \mathrm{q} 26.3$.

To investigate the presence of interchromosomal effect, triple-color FISH was performed using the second probe mixture, which consists of commercial satellite (DNA) probes from Vysis, including chromosomes 18, X and $\mathrm{Y}$ (CEP 18, Spectrum Blue/CEP X, Spectrum Green/CEP Y, Spectrum Red). Post-hybridization washes included $2 \mathrm{~min}$ in $0.4 \times S S C / 0.3 \% \mathrm{NP}-40(\mathrm{pH}=7)$ at $72{ }^{\circ} \mathrm{C}$, followed by $1 \mathrm{~min}$ in $2 \mathrm{xSSC} / 0.1 \% \mathrm{NP}-40(\mathrm{pH}=7)$ at room temperature. Slides were covered with DAPI II (Vysis). Only intact spermatozoa bearing a similar degree of decondensation and clear hybridization signals were scored. All disrupted or overlapping spermatozoa were excluded from analysis. Slides with hybridization efficiency of $99 \%$ and more were analyzed. 1,000 sperm nuclei were analyzed per patient.

\section{Array comparative genomic hybridization (aCGH)}

Array comparative genomic hybridization $(\mathrm{aCGH})$ has been introduced in clinical diagnosis to rapidly detect genomewide gains and losses with higher resolution [4]. It is a high throughput method which detects copy number changes to a resolution of even as low as $1 \mathrm{~Kb}$. For aCGH analysis, DNA was extracted from peripheral blood [5]. aCGH analysis was performed on using the 


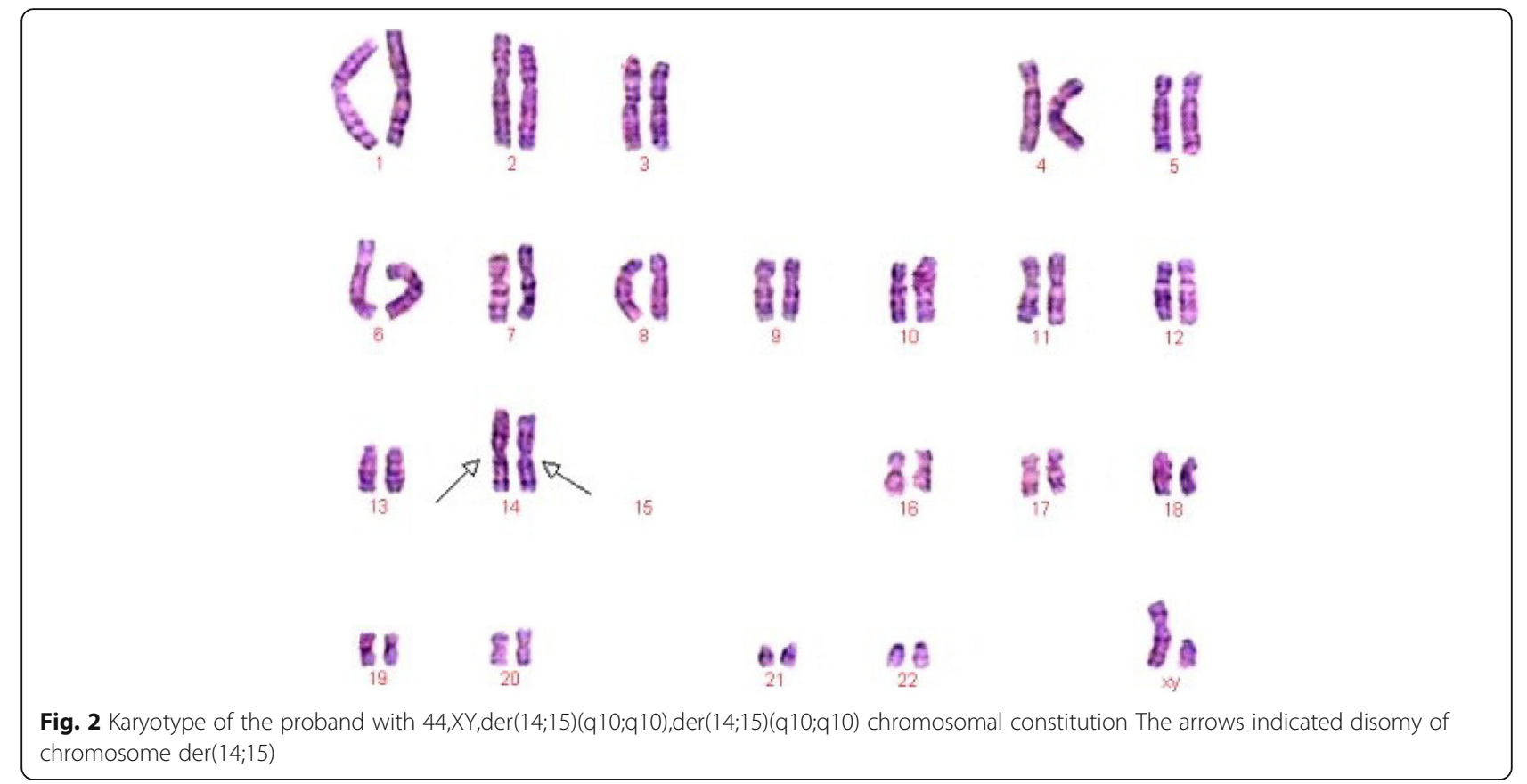

$8 \times 60 \mathrm{~K}$ commercial arrays (Agilent Technologies, Santa Clara, CA, USA).

\section{Data analysis}

The statistical analysis was performed using the SAS system (2002-2003, SAS Institute Inc., Cary, NC). Chi-squared test was used for meiotic segregation analysis. A probability value of less than $0.05(P<0.05)$ was considered to be statistically significant.

\section{Results}

The karyotype analysis was performed on four members in the family and detected two different karyotypes of Robertsonian translocation. The results are shown in below. III-1 and III-2 were heterozygous while IV-1 was homozygous.

$$
\begin{aligned}
& \text { III-1: 45,XY,der(14;15)(q10;q10) } \\
& \text { III-2: 45,XX,der(14;15)(q10;q10) } \\
& \text { IV-1: 44,XY,der(14;15)(q10;q10), } \operatorname{der}(14 ; 15)(\mathrm{q} 10 ; \mathrm{q} 10) \\
& \text { IV-2: 46,XX }
\end{aligned}
$$

Because the patient (IV-1) has a symptom of apathy, we referred him to see a psychiatrist for a thorough mental status examination. The patient had no psychiatric history and remained cooperative during the psychiatric interview. His thought processes were linear, logical, and consistently goal directed and his judgment was intact. Therefore, no psychiatric symptoms were observed. In addition, neurology consultation revealed no focal neurological deficits. The results of Craniocerebral Computed Tomography (CT) and lumbar puncture (LP) were normal. Electroencephalography (EEG) showed no signs of seizure activity.

The aCGH analysis on IV-1 detected a $2.03 \mathrm{Mb}$ duplication in the region of q11.1-q11.2 on chromosome 15 (Fig. 3). According to the Copy number variation database (CNVdb), this duplication is a chromosomal polymorphism in normal population. Because IV-1 is a is Homologous Robertsonian translocation carrier and his two derivative chromosomes came from the same Robertsonian translocation carrier, we speculated that each of the two derivative chromosomes should have a $2.03 \mathrm{Mb}$ duplication in the region of q11.1-q11.2 on chromosome 15.

To analysis the sperm of III-1 and IV-1, semen samples from the two different Robertsonian translocation carriers (III-1 and IV-1) were assessed for volume, concentration and motility according to the World Health Organization criteria. The volume, concentration and mobility of sperm in III-1 are significantly reduced due to his old age. In contrast, they are all normal in IV-1 (Table 1). We further analyzed the meiotic segregation patterns in these two different Robertsonian translocation carriers. In the heterozygote Robertsonian translocation carrier (III-1), the frequency of normal/balanced spermatozoa resulting from alternate segregation was $79.9 \%$ while the frequency of unbalanced spermatozoa resulting from adjacent segregation was $20.1 \%$, which is significantly higher than normal population [5-8] (Table 2). In contrast, in the homozygote Robertsonian translocation (IV-1), the frequency of balanced spermatozoa was $99.7 \%$ while the frequency of unbalanced spermatozoa was only $0.3 \%$, which is similar to normal 

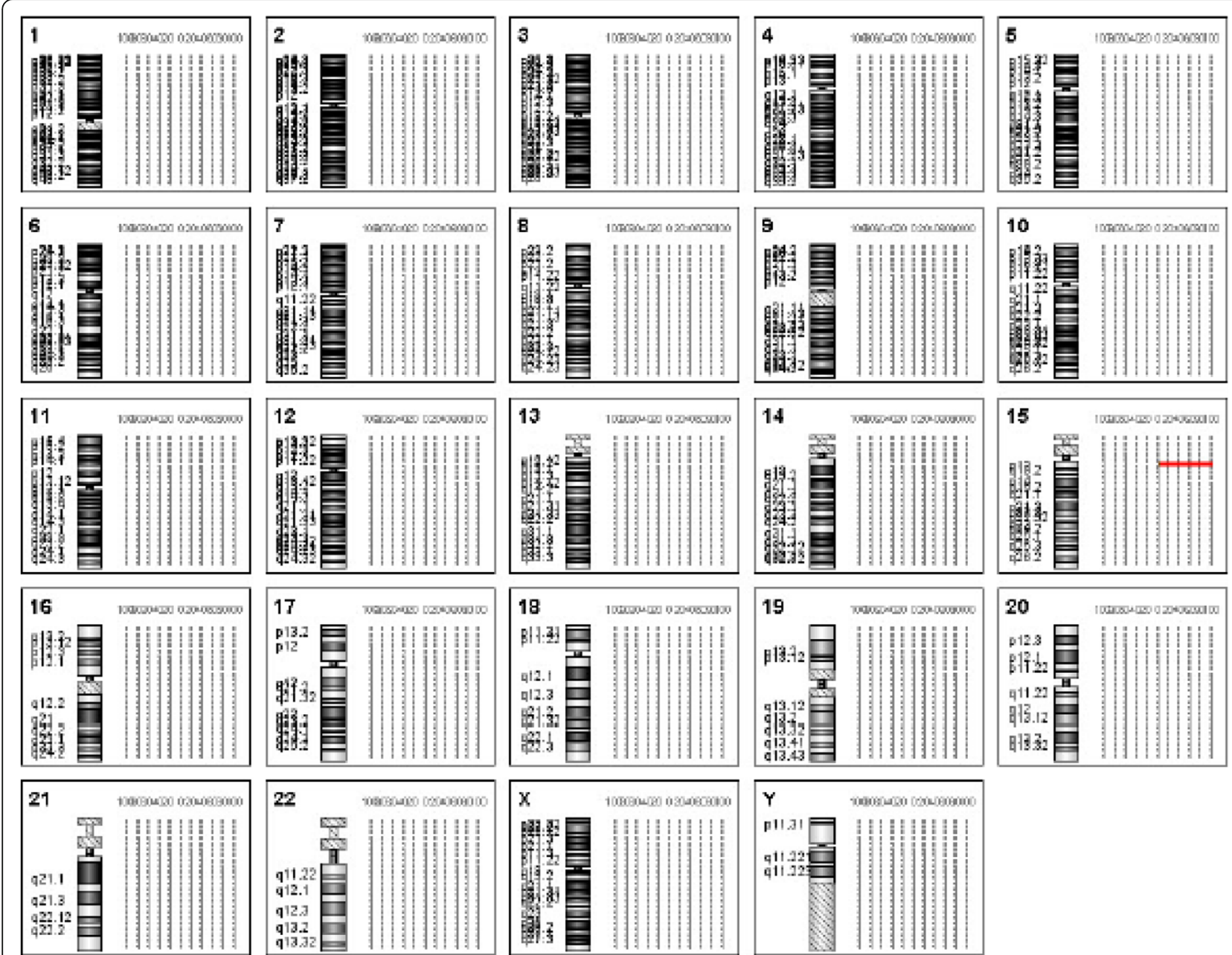

Fig. 3 aCGH analysis of IV-1 revealed a 2.03 Mb duplication at chromosome 15 q11.1-q11.2 (arr 15q11.1q11.2(20,481,702-22,509,254)x4)

populations [5-8] (Table 2). These data demonstrated there nearly all sperm of Robertsonian translocation homozygotes were balanced while those of heterozygotes had a percentage of unbalanced. In addition, chromosome analysis of IV-1's sperm showed either a 22,X,der $(14 ; 15)$ karyotype or $22, \mathrm{Y}, \mathrm{der}(14 ; 15)$ karyotype supporting our speculation. As shown in Fig. 4, the arrow pointed a sperm with four fluorescence signals (two red and two green) suggesting this sperm has two derivative chromosomes of der(14;15). These data suggest a potential mechanism of speciation in humans via Robertsonian translocation.

Table 1 Cytogenetic and spermiologic results of III-1 and IV-1

\begin{tabular}{llll}
\hline Patient & Age (years) & $\begin{array}{l}\text { Sperm concentration } \\
\left(\times 10^{6} / \mathrm{ml}\right)\end{array}$ & Mobility $(\mathrm{a}+\mathrm{b})(\%)$ \\
\hline III-1 & 48 & 13 & 22 \\
IV-1 & 25 & 56 & 53 \\
\hline
\end{tabular}

The influence of translocated chromosomes on the synapses and disjunction of other chromosomes is called an interchromosomal effect (ICE) [9]. To determine whether ICE was present, the nullisomy, disomy and diploid rates for chromosomes $18, \mathrm{X}$ and $\mathrm{Y}$ were analyzed in both Robertsonian translocation carriers (Table 3). In the heterozygote Robertsonian translocation carrier (III-1), there was a significant increase in the rate of sex chromosome aneuploidy and diploid. In contrast, in the homozygote Robertsonian translocation (IV-1), the incidence of spermatozoa with nullisomy, disomy and diploid for the sex chromosomes remained similar to normal populations (Table 3) [5-8]. This data suggest that ICE only presents in certain Robertsonian translocation carriers.

\section{Discussion}

Rob translocation carries are phenotypically normal since they involve loss only of short arm material. Many 
Table 2 The number of spermatozoa scored, the alternate mode of segregation, incidence of sperm nullisomy, disomy and 3:0/diploid for the chromosomes involved in the Rob translocation in two Rob translocation carriers

\begin{tabular}{lll}
\hline Segregation modes & III-1 & IV-1 \\
\hline normal or balanced & 799 & 997 \\
nullisomy 14 & 41 & 2 \\
disomy 14 & 49 & 0 \\
nullisomy 15 & 55 & 0 \\
disomy 15 & 45 & 0 \\
3:0 or diploid & 11 & 1 \\
Total & 1000 & 1000 \\
\hline
\end{tabular}

such rearrangments in natural populations of different species are known. Robertsonian rearrangments can increase polymorphisms in a species, provide material for natural selection and even lead to speciation [10, 11]. It has been argued that the types of chromosome rearrangments that occur as polymorphisms or as fixed permanent heterozygotes invariably involve meta- or submetacentric chromosomes. Those that distinguish species and serve to isolate these species involve telocentric or acrocentric chromosomes, which are self-sterilizing [12]. In our study, IV-1, who is a homologous Robertsonian translocation carrier, was healthy and had a balanced chromosomal complement. Assessment of a semen sample from showed normal sperm morphology and motility. More importantly, $99.7 \%$ of his sperm are normal or balanced (Table 2) and there was no interchromosomal effect (Table 3).

Multicolor FISH on decondensed sperm nuclei allows for a rapid analysis of meiotic segregation in sperm of translocation carriers, providing information on the exact amount of normal/balanced sperm [13]. Such information is undoubtedly important not only for basic cytogenetic research but also for reproductive counseling

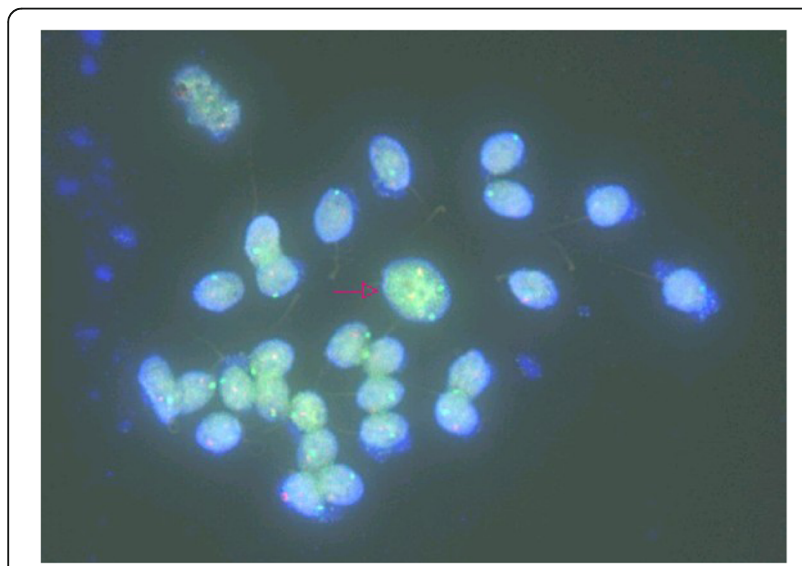

Fig. 4 Sperm cells after hybridization with TelVysion 14q32.33 (Spectrum Red) and TelVysion 15q26.3 (Spectrum Green). The sperm with red arrow may be a diploid sperm
Table 3 Incidence of sperm nullisomy, disomy and diploid for chromosomes 18, $\mathrm{X}$ and $\mathrm{Y}$ in two Rob translocation carriers

\begin{tabular}{lll}
\hline Segregation modes & III-1 & IV-1 \\
\hline normal or balanced & 949 & 994 \\
nullisomy 18 & 3 & 0 \\
disomy 18 & 2 & 1 \\
nullisomy Sex chromosome & 15 & 2 \\
disomy Sex chromosome & 10 & 3 \\
3:0 or diploid & 21 & 0 \\
Total & 1000 & 1000 \\
\hline
\end{tabular}

of Robertsonian translocation carriers. $\mathrm{t}(14 ; 15)$ are rare Robertsonian translocations. Our data confirmed previous studies showing that alternate segregation was more frequently than adjacent segregations in Robertsonian translocations carriers $[5,8]$. However, the meiotic segregation patterns and interchromosomal effects in two Robertsonian translocation carriers are different (Table 2). These findings are consistent with previously published data [14-16]. The high prevalence of the alternate segregation is presumably due to cis-configuration of the trivalent during meiosis, which favors an alternate segregation in all Robertsonian translocations [2]. Although a Robertsonian translocation carrier has a full genetic complement, their productive fitness is reduced due to high probability of genetically imbalanced gametes.

The interchromosomal effect could be explained by the formation of heterosynapses between chromosomes involved in the translocation and the sex vesicle, which could also involve other chromosomes [17]. ICE remains controversial in the literature. Some publications have indicated its presence with Robertsonian translocation $[18,19]$ while others demonstrated no evidence of this phenomenon $[4,20]$. In our study, we observed higher incidence of aneuploidy for sex chromosomes in spermatozoa in the heterozygote Robertsonian translocation but not the homozygote Robertsonian translocation suggesting that ICE only presents in some Robertsonian translocation carriers.

Robertsonian rearrangements are common chromosomal changes that can lead to rapid and efficient reproductive isolation between karyotypically similar populations, especially when many Robertsonian metacentric chromosomes display monobrachial homologies [10]. Homozygous Robertsonian translocations have reported by several groups [21-23] and systemically reviewed by the O'Neill group [24]. Dallapiccola et al. [21] reported a fetus with two $\mathrm{t}(14 ; 21)$ chromosomes. The related parents were heterozygous for the same translocation. Martinez et al. [22] reported three adult siblings homozygous for $t(13 ; 14)$. Their parents were first-cousins and both were heterozygous carriers. Rajangam et al. [23] found a unique DS karyotype 45, 
$\mathrm{XY}$, der(14;21)pat, der(14;21)mat, +21mat. Although translocation heterozygosity is associated with meiotic disturbances, which will lead to infertility and subfecundity, it should not have any effect on meiosis. Here we reported a man with a 44,XY,der(14;15)(q10;q10),der(14;15)(q10;q10) karyotype. Robertsonian translocation may provide material for evolution. Long term isolation of a group of individuals who are homozygous for a particular Robertsonian translocation chromosome could theoretically lead to the establishment of a new human subspecies having a full genetic complement in 44 chromosomes. Although karyotypic differences between species have long been recognized, the question of whether these mutations play a causal role in speciation remains unanswered [25-27]. In our study, all sperm of Robertsonian translocation homozygotes were balanced while those of heterozygotes had a percentage of unbalanced ones implicating of a potential of the homozygosity of Robertsonian translocations for speciation.

\section{Conclusions}

To conclude, in this study we reported a previously described Robertsonian translocation karyotype. The homozygosity of Robertsonian translocation for speciation may be a potential mechanism of speciation in Humans.

\section{Abbreviations}

aCGH, Array comparative genomic hybridization; CT, Craniocerebral Computed Tomography; EEG, Electroencephalography; FISH, Fluorescence in situ hybridization; ICE, interchromosomal effect; LP, lumbar puncture

\section{Funding}

This work is supported by a scientific research and technology development grant of Guangxi province (\#1598012-35)

\section{Availability of data and materials}

The dataset(s) supporting the conclusions of this article are available in the folder named raw data for the manuscript at https://www.dropbox.com/sh/ izfb6bdmyz43gz7/AAB4nVTovhquFbSqA3kqtTtHa?dl=0

\section{Authors' contributions}

B.W., J.S. and L.S designed the study. B.W. and J.S. analyzed the results and wrote the manuscript. L.S. revised the manuscript. X. L. perfomred the sperm FISH. S. X. participated in genetic counseling. N. L. and Z. L. performed array CGH anaysis. Y. Y. and W.W. performed chromosome analysis. H. R. perforemed psychiatric interview. All authors read and approved the final manuscript.

\section{Competing interests}

The authors declare that they have no competing interests.

\section{Ethics approval and consent to participate}

The affected patient were informed about the study, and written informed consent, approved by the Ethical committee of Hubei Maternal and Child Health Hospital, was obtained.

\section{Author details}

${ }^{1}$ Genetics Laboratory, Hubei Maternal and Child Health Hospital, Wuhan, Hubei, People's Republic of China. ${ }^{2}$ Department of gastroenterology, Peking University Shenzhen Hospital, Shenzhen, Guangdong, People's Republic of China. ${ }^{3}$ Laboratory of Medical Genetics, Qinzhou Maternal and Child Health Care Hospital, Guangxi, People's Republic of China. ${ }^{4}$ Shenzhen mental health center, Shenzhen Kangning Hospital, Shenzhen, Guangdong, People's Republic of China.
Received: 14 January 2016 Accepted: 3 June 2016

Published online: 18 June 2016

\section{References}

1. Therman E, Susman B, Denniston C. The nonrandom participation of human acrocentric chromosomes in Robertsonian translocations. Ann Hum Genet. 1989:53:49-65.

2. Luciani JM, Guichaoua MR, Mattei A, Morazzani MR. Pachytene analysis of a man with a 13q;14q translocation and infertility. Cytogenet Cel Genet. 1984;38:14-22

3. Cassuto NG, Le Foll N, Chantot-Bastaraud S, Balet R, Bouret D, Rouen A et al. Sperm fluorescence in situ hybridization study in nine men carrying a Robertsonian or a reciprocal translocation: relationship between segregation modes and high-magnification sperm morphology examination. Fertil Steri. 2011:96:826-32.

4. ACOG Committee Opinion No. 446: array comparative genomic hybridization in prenatal diagnosis. Obstet Gynecol. 2009;114(5):1161-3.

5. Ferfouri F, Selva J, Boitrelle F, Gomes DM, Torre A, Albert M, et al. The chromosomal risk in sperm from heterozygous Robertsonian translocation carriers is related to the sperm count and the translocation type. Fertil Steril. 2011;96:1337-43.

6. Mahjoub M, Mehdi M, Brahem S, Elghezal H, Ibala S, Saad A. Chromosomal segregation in spermatozoa of five Robertsonian translocation carriers t(13;14). J Assist Reprod Genet. 2011;28:607-13.

7. Rouen A, Pyram K, Pollet-Villard X, Hyon C, Dorna M, Marques S, et al. Simultaneous cell by cell study of both DNA fragmentation and chromosomal segregation in spermatozoa from chromosomal rearrangement carriers. J Assist Reprod Genet. 2013. doi:10.1007/s10815012-9915-7.

8. Machev N, Gosset P, Warter S, Treger M, Schillinger M, Viville S. Fluorescence in situ hybridization sperm analysis of six translocation carriers provides evidence of an interchromosomal effect. Fertility and Sterility. 2005:84(2):365-73.

9. Douet-Guilbert N, Bris MJ, Amice V, Marchetti C, Delobel B, Amice J, et al. Interchromosomal effect in sperm of males with translocations: report of 6 cases and review of the literature. Int J Androl. 2005;28:372-9.

10. Britton-Davidian J, Catalan J, da Draca RM, et al. Rapid chromosomal evolution in island mice. Nature. 2000;403:158

11. Wang W, Lan H. Rapid and parallel chromosomal number reductions in muntjac deer inferred from mitochondrial DNA phylogeny. Mol Biol Evol. 2000;17:1326-33.

12. John B. Chromosome change and evolutionary change: A critique. In: Atchley WR, Woodruff DS, editors. Evolution and specification: Essays in honor of M.J.D. White, Chapter 2. London, New York: Cambridge University; 1981

13. Benet J, Oliver-Bonet M, Cifuentes P, Templado C, Navarro J. Segregation of chromosomes in sperm of reciprocal translocation carriers: a review. Cytogenet Genome Res. 2005;111:281-90.

14. Roux C, Tripogney C, Morel F, Joanne C, Fellmann F, Clavequin MC, et al. Segregation of chromosomes in sperm of Robertsonian translocation carriers. Cytogenet Genome Res. 2005;111:291-6.

15. Rives N, Ravel C, Duchesne V, Siffroi JP, Mousset-Simeon N, Mace B. Molecular cytogenetics analysis with whole chromosome paint probes of sperm nuclei from a $(13 ; 15)$ Robertsonian translocation carrier. J Hum Genet. 2005:50:360-4.

16. Anahory T, Hamamah S, Andreo B, Hedon B, Claustres M, Sarda P, et al. Sperm segregation analysis of a $(13 ; 22)$ Robertsonian translocation carrier by FISH: a comparison of locus-specific probe and whole chromosome painting. Hum Reprod. 2005:20:1850-4.

17. Lindenbaum $\mathrm{RH}$, Hulten $\mathrm{M}, \mathrm{McDermott} \mathrm{A}$, Seabright $\mathrm{M}$. The prevalence of translocations in parents of children with regular trisomy 21: a possible interchromosomal effect? J Med Genet. 1985;22:24-8.

18. Anton E, Blanco J, Vidal F. Meiotic behavior of three D;G Robertsonian translocations: segregation and interchromosomal effect. J Hum Genet. 2010;55:541-54.

19. Ogur G, Van Assche E, Vegetti W, Verheyen G, Tournaye H, Bonduelle M, et al. Chromosomal segregation in spermatozoa of 14 Robertsonian translocation carriers. Mol Hum Reprod. 2006:12:209-15.

20. Anton E, Vidal F, Blanco J. Role of sperm FISH studies in the genetic reproductive advice of structural reorganization carriers. Hum Reprod. 2007;22:2088-92. 
21. Dallapiccola B, Ferranti G, Altissimi D, Colloridi F, Paesano R. First-trimester prenatal diagnosis of homozygous $(14 ; 21)$ translocation in a fetus with 44 chromosomes. Prenat Diagn. 1989;9:555-8.

22. Martinez-Castro P, Ramos MC, Rey JA, et al. Homozygosity for a Robertsonian translocation (13q14q) in three offspring of heterozygous parents. Cytogenet Cell Genet. 1984;38:310-2.

23. Rajangam S, Michaelis RC, Velagaleti GV, Lincoln S, Hegde S, Lewin S, Tarleton J, Thomas IM , Tharapel AT. Down Syndrome With Biparental Inheritance of der(14q21q) and Maternally Derived Trisomy 21: Confirmation by Fluorescent In Situ Hybridization and Microsatellite Polymorphism Analysis. Am J Med Genet Part A. 1997;70:43-7.

24. O'Neill ID. Homozygosity for constitutional chromosomal rearrangements: a systematic review with reference to origin, ascertainment and phenotype. J Hum Genet. 2010;55(9):559-64.

25. Rieseberg LH. Chromosomal rearrangements and speciation. Trends Ecol Evol. 2001;16(7):351-8. doi:10.1016/50169-5347(01)02187-5.

26. Heng HH. Elimination of altered karyotypes by sexual reproduction preserves species identity. Genome. 2007;50(5):517-24.

27. Horne SD, Chowdhury SK, Heng HH. Stress, genomic adaptation, and the evolutionary trade-off. Front Genet. 2014;134(9):2074-87.

Submit your next manuscript to BioMed Central and we will help you at every step:

- We accept pre-submission inquiries

- Our selector tool helps you to find the most relevant journal

- We provide round the clock customer support

- Convenient online submission

- Thorough peer review

- Inclusion in PubMed and all major indexing services

- Maximum visibility for your research

Submit your manuscript at www.biomedcentral.com/submit
Biomed Central 